\title{
Anatomic shoulder arthroplasty: an update on indications, technique, results and complication rates
}

\author{
LORENZO MATTEI, STEFANO MORTERA, CHIARA ARRIGONI, FILIPPO CASTOLDI
}

I and II Clinica Ortopedica Traumatologica, CTO, Città della Salute e della Scienza, University of Turin, Turin, Italy

\begin{abstract}
A shoulder replacement is indicated in patients affected by glenohumeral arthropathy with severely reduced range of motion, persistent pain, especially at night, and loss of strength. There is much discussion in the scientific community about the prosthetic options for these cases: hemiarthroplasty, anatomic total shoulder arthroplasty, and reverse total shoulder arthroplasty. We analyzed the indications for, results of, and complications associated with this kind of surgery, focusing on anatomic arthroplasty and on the concept of modularity.
\end{abstract}

Key Words: shoulder arthroplasty, indications, complications, results, modularity, shoulder fractures.

\section{Introduction}

The first shoulder prosthesis was implanted in 1893 by the French surgeon Péan. The patient was a baker affected by tubercular arthritis. For a short time, his range of motion was found to be improved, but the infection recurred and the prosthesis was removed two years after its implantation (1).

The development of modern shoulder arthroplasty dates back to 1951, when Charles Neer used vitallium prostheses to treat proximal humerus fractures. Before long, these prostheses were also being used in fracture sequelae and osteonecrosis. In an article published in 1970, Neer reported a $90 \%$ rate of good

\footnotetext{
Corresponding Author:

Lorenzo Mattei, MD

I and II Clinica Ortopedica Traumatologica, CTO, Città

della Salute e della Scienza, University of Turin

Via Gianfranco Zuretti, 29

10126 Turin, Italy

E-mail: lorenzo.mattei87@gmail.com
}

or successful outcomes in 43 patients (2); he progressively modified the prosthesis, to make it suitable for treating glenohumeral osteoarthritis (OA), and added a polyethylene glenoid component.

During the same period, many groups in Europe and in the United States, transferring the constrained hip prosthesis concepts to the shoulder, developed new prostheses. However, in the 1970s and 1980s, the use of constrained shoulder prostheses produced unacceptable glenoid loosening rates and poor clinical outcomes, leading to the misconception, which is still prevalent today, that shoulder arthroplasty is not effective. Conversely, in a 1982 study of 250 total shoulder arthroplasties, performed for a variety of inflammatory degenerative or post-traumatic conditions, Neer et al. reported a very high satisfaction rate and excellent functional outcomes in patients who had no rotator cuff lesions (3). In this study, Neer classified the indications for shoulder arthroplasty and evaluated the outcomes for each indication. This classification remains relevant today.

In those years, Neer's monoblock prosthesis was replicated and improved by the introduction of a modular prosthesis having two components (a stem and a head) that were each available in various sizes to enable adjustment to the patient's geometry. The aim was to overcome the limitations related to the anatomical variations of the humerus, the space available for the joint, and the diameters of the medullary canal of the humerus. With regard to the glenoid component, some models offered fixing without cement, but with screws and porous metal coatings to support the polyethylene. Two major problems were encountered. First, the prosthetic head was often poorly positioned in both the vertical and the horizontal planes. Second, the head was frequently oversized. Despite their modularity, second-generation prostheses did not allow replication of the proximal humeral anatomy and even created new problems. Thus, in the 1990s, techniques allo- 
wing accurate computerized measurements of proximal humerus geometry and of different sizes of humerus were developed. These measurements influenced the concepts underlying anatomic reconstruction of the proximal humerus (4), leading to the development of third-generation prostheses, adaptable to the individual characteristics of each patient.

Nowadays, the head components are available in several sizes and thicknesses, and the position of the head relative to the shaft can be adjusted. By resecting the humeral head at the anatomic neck and using an implant that can be constructed to match the original head retroversion, inclination, medial and posterior offset, and to have a depth identical to that of the native humeral head, the individual lever arms of the rotator cuff muscles are restored. Today, this "anatomic reconstruction" of the joint results in normal kinematics and kinetics (5).

\section{Anatomic shoulder arthroplasty in osteoarthritis}

A shoulder replacement is indicated in patients affected by glenohumeral arthropathy with severely reduced range of motion, persistent pain, especially at night, and loss of strength.

There is much discussion in the scientific community about the prosthetic options for these cases: hemiarthroplasty, anatomic total shoulder arthroplasty (TSA), and reverse total shoulder arthroplasty (RTSA).

The indications for TSA are: severe primary OA or secondary OA caused by instability, osteonecrosis, inflammatory joint diseases and, in some cases, complex proximal humeral fractures.

Instead, a TSA cannot be implanted when the soft tissues are no longer reparable or there is a severe posterior glenoid deficiency, due to degenerative pathologies, trauma or previous surgery.

The presence of a functioning rotator cuff is mandatory to obtain a good result. In cases with a reparable cuff tear, TSA remains a valid option (6).

If $\mathrm{OA}$ is associated with recurrent instability or with excessive capsular laxity, it is possible to associate the appropriate soft-tissue balancing, or to use a larger head that will increase joint stability, by increasing capsular tension (7).

In patients younger than 50 , with rotator cuff insufficiency, an intact coracoacromial arch and adequate abduction strength provided by the deltoid muscle, it is possible to perform anatomical replacement or head resurfacing $(7,8)$.

Reaming of the glenoid is essential in TSA. The version of the glenoid must be properly corrected, considering the central axis of the glenoid. It is essential to restore the glenoid arc, which is necessary to ensure joint stability, cuff balance and deltoid forces. Failure to do this results in a risk of premature loosening of the glenoid component (rocking horse effect) (9).

Axial images of a CT scan are useful for evaluating the morphology of the glenoid surface and measuring the depth of the glenoid; the glenoid depth must be at least $15 \mathrm{~mm}$ in order for the bone stock to be considered sufficient for the implant (10).

In $\mathrm{OA}$, focal load concentration and progressive joint surface deformation typically cause posterior glenoid wear, resulting in a "biconcave" glenoid (11). When there is alteration of the glenoid anatomy, normal load transfer and stability cannot be restored by hemiarthroplasty; indeed, the use of this technique in the presence of glenoid wear is associated with poor outcomes (12). Biologic glenoid resurfacing procedures, such as the interposition of fascial, capsular or meniscal allograft tissue, can be considered in order to address the problem of glenoid wear. The important questions about these procedures, which remain unanswered, concern the durability of the interposed material and whether or not the material becomes fixed to the glenoid bone. In the presence of severe wear, it is, nevertheless, possible to implant a prosthetic glenoid component, but the risk of mobilization in the medium and long term is substantially increased $(13,14)$.

In patients with glenoid dysplasia, there are different treatment options: bone grafting of the deficient region in conjunction with the placement of a glenoid component; bone grafting or glenoid osteotomy alone in conjunction with a hemiarthroplasty; or use of a glenoid component with metal augments that substitute for the deficient bone (15). Other Authors have adapted their choice to the glenoid abnormality and chosen hemiarthroplasties when the bone stock was not sufficient (10).

Correct reaming of a degenerative glenoid can be a difficult procedure in TSA (16-22). Mild deformities may be corrected by eccentric reaming. However, severe deformities have a high rate of failure at midterm follow-up. A biconcave glenoid $(23,24)$ is a difficult situation from the perspective of reconstruction; the use of a TSA in this situation is associated 
with high failure rates due to early glenoid loosening or recurrent posterior instability. When posterior humeral head subluxation is greater than $80 \%$ or retroversion of the replaced glenoid is greater than $27^{\circ}$, an unacceptably high rate of complications has been observed. When posterior erosion cannot be adequately corrected with eccentric reaming, particularly in older patients, RTSA may be a viable alternative to unconstrained TSA.

Static posterior subluxation of the humeral head (PSH) (subluxation index of $>65 \%$ ) is often associated with primary glenohumeral OA or secondary to anterior shoulder repair (which can lead to a loss of glenohumeral external rotation without any abnormality of glenoid version) and may persist following TSA. There is no correlation between the presence or absence of static PSH and a specific range of glenoid or humeral version, or altered capsulolabral biomechanics, the condition being primary or secondary to prior anterior stabilization procedures.

Preoperative PSH was recently shown to be the primary contributor to early glenoid loosening in a series of metal-backed uncemented glenoid prostheses (25). Nevertheless, excellent clinical results can be obtained with TSA in OA associated with PSH. The combination of a generous capsulolabral release, adequate mobilization of the subscapularis musculotendinous unit, attempted correction of glenoid version by eccentric reaming, and reproduction of native humeral retrotorsion corrects static PSH in the vast majority of cases (26). However, postoperative PSH is likely to lead to rim loading of the glenoid component, accelerated polyethylene wear and glenoid loosening. It is possible that PSH which persists postoperatively may be due to overtensioning of the subscapularis or excessive rotator interval closure.

\section{Fracture shoulder arthroplasty}

When treating three or four-part fractures of the proximal humerus, surgeons in the United States and Europe find it difficult to replicate the $90 \%$ rate of good or excellent outcomes reported by Neer with his original prosthesis. The main problem concerns the healing of the tuberosities around the prosthesis, with most case series showing non-union, resorption or displacement of the greater tuberosity. Therefore, can anatomic shoulder prosthesis still be considered a good surgical solution?
Although shoulder hemiarthroplasty is technically demanding in the presence of fractures, with difficulty often encountered in achieving the correct humeral stem height and appropriate retroversion of the prosthesis (27), it has traditionally been considered the "gold standard" for fractures in which adequate open reduction and internal fixation (ORIF) cannot be achieved or is contraindicated. Shoulder hemiarthroplasty is particularly indicated in cases in which avascular necrosis (AVN) of the humeral head seems inevitable. According to Hertel et al. (28), the predictors of AVN of the humeral head are the integrity of the medial hinge, the length of the dorsomedial metaphyseal extension of the head fracture (calcar length), and the fracture type. In the elderly, fracture-dislocations, disruption of the medial periosteal hinge, and non-reconstructable head-split or humeral head impression fractures should be managed by hemiarthroplasty (29). Also, when ORIF cannot provide a stable anatomically reduced fracture in young individuals without rotator cuff tears or comminuted fracture and with good bone quality, a humeral head replacement may be considered. Repair and healing of the greater and lesser tuberosities are among the factors that have the greatest influence on the fate of a shoulder hemiarthroplasty (30). Evolutions in tuberosity fixation techniques and in implant design (the development of fracture-specific humeral stems) represent attempts to rectify some of the early problems involving tuberosity healing and implant malpositioning.

In acute fractures, shoulder hemiarthroplasty is usually preferred to TSA given that, in these cases, significant glenoid cartilage damage has not yet developed (31). Additional reasons for choosing shoulder hemiarthroplasty are the avoidance of glenoid component complications (e.g., loosening, polyethylene wear) and the feasibility of secondary conversion to TSA if required (32). Modular prostheses facilitate restoration of head-tuberosity-shaft relationships by variable adjustment of humeral height, offset and retroversion (33).

\section{Modularity}

The results of studies involving shoulder hemiarthroplasty as a treatment for humeral head fractures are not homogeneous, with many patients reporting pain relief with varying functional results. However, the survival rate reported in recent studies ranges from 50 to $80 \%$ at medium-term follow-up (34). Implant failu- 
re is frequently due to tuberosity resorption, tuberosity non-union and rotator cuff deficiency (30).

Successful management of failures continues to be a challenge. Revision of the implant to RTSA may be one possible option (35). Generally, revision surgery is highly demanding due to the need to remove the humeral stem. The presence of the cement or of marked ingrowth of a cementless component makes the revision procedure difficult and carries a risk of humerus fracture (36). In fact, a well-fixed humeral implant constitutes an additional source of difficulty when, as in most cases, the reason for the revision is a problem with the glenoid component or the presence of a rotator cuff failure. The use of a modular shoulder system allows revision of an anatomic prosthesis to RTSA with a reduced complication rate.

Modularity is provided by a range of humeral heads of increasing size. A single head thickness is used for each diameter so that the anatomical variations encountered can be better matched. It is important that the thickness and the diameter of the articular surface are always respected so that the muscle-ligament balance of the shoulder can be recovered and the different curvature radii of the head-glenoid complex can be maintained (37).

\section{Results}

Nowadays, unconstrained TSA is widely used to treat glenohumeral $\mathrm{OA}$, rheumatoid arthritis and osteonecrosis, with good and reproducible results $(38,39)$.

The use of prostheses seems to have a less predictable outcome in complex humeral fractures as compared with cuff tear arthropathy.

Usually the patients are pain free, but most have some difficulties in activities of daily living due to a limited range of motion. These poor results have led to the development of specific implants, dedicated prostheses for fractures and semi-constrained reverse geometry designs (RTSA) for cuff tear arthropathy.

The question of whether TSA or shoulder hemiarthroplasty is better for the treatment of $\mathrm{OA}$ is one that is often debated within the scientific community. In our experience, better results are obtained with TSA. This idea is confirmed by a multicenter study showing better results (greater patient satisfaction and wider range of motion) with TSA (18).

Resurfacing hemiarthroplasty is associated with a poorer outcome and a higher risk of revision than pre- viously assumed, especially in patients under the age of 55 years. It seems that TSA should be preferred for the treatment of glenohumeral OA (40).

A literature review analyzing the results of TSA and hemiarthroplasty for glenohumeral OA was published by van den Bekerom et al. (41). Pain relief, patient satisfaction and significant improvement in function were observed at two to seven years of follow-up in more than $90 \%$ of cases treated for primary OA with either hemiarthroplasty or TSA. In patients with minor thinning or small tears of the rotator cuff that were adequately addressed at surgery, these conditions were not found to affect pain, patient satisfaction or range of motion. The rate of revisions, performed for any reason, was higher in the hemiarthroplasty group $(13 \%)$ than in the TSA group $(7 \%)(p<0.001)$, and no difference was found between revisions of the humeral or glenoid component. A trend towards a higher rate of complications (of any kind) was found in the TSA group $(12 \%)$ compared with the hemiarthroplasty group $(8 \%)$. In both groups the range of motion improved. The Authors concluded that TSA resulted in less need for revision surgery, but had a higher complication rate. Similar results were previously found by Singh et al. (39).

\section{Complications}

Major complications in shoulder prostheses are: periprosthetic fractures, infections, instability, rotator cuff lesions, loosening of the glenoid component and neurological injuries.

The reported prevalence of periprosthetic fractures is between 1.6 and $2.3 \%$ (42). Potential etiologies include: osteopenia, cortical thinning due to osteolysis, excessive reaming of the humeral cortex during preparation of the diaphyseal channel and eccentric placement of the humeral component (43). These fractures are classified according to a classification system described by Wright and Cofield, which is based on the location of the fracture in relation to the tip of the prosthesis. The literature indicates that this classification helps to predict the outcome of fractures and is also useful for choosing the right treatment (42). It is also important, from this perspective, to distinguish between the presence and absence of humeral component loosening.

The reported prevalence of infections is between 0 and $4 \%(44,45)$. The effect of a deep infection at the implant site may result in patients needing to undergo 
protracted antibiotic therapies and urgent revision surgery, which is normally more difficult for the surgeon and more invasive for the patient.

In general, every area of osteolysis must be considered a site of infection until this diagnosis can be excluded. Instability can be superior, anterior and posterior. Superior instability is generally associated with a rotator cuff lesion or with a lesion of the coracoacromial ligament. In these cases it can be treated by repairing the rotator cuff (in young patients) or by converting to a reverse prosthesis (in the elderly).

The reported prevalence of anterior instability is between 0.9 and $1.8 \%$, while that of posterior instability is $1 \%(46,47)$. Anterior instability is due to a subscapularis lesion or to excessive stem/glenoid anteversion. In these cases, subscapularis repair, change of the component or pectoralis major transposition do not provide satisfactory results $(48,49)$, and the only solution likely to be successful is conversion to TRSA. Posterior instability is due to excessive posterior capsule laxity or to excessive stem/glenoid retroversion. Sanchez-Sotelo et al. (48) observed that, when instability is addressed and corrected, outcome is better in patients with posterior instability than in those with anterior instability.

Rotator cuff tears are reported with an incidence between 1.3 and $7.8 \%(47,50-53)$. The subscapularis tendon is the mostinjured. Often the lesion is asymptomatic, but it results in a loss of strength. To avoid this complication, care must be taken during surgical mobilization of tendons. It is necessary to perform careful release of adhesions under the coracoid and under the glenohumeral ligaments. In the case of traumatic lesions, it is possible, in young patients, to repair the tendons with good results. In older patients, in whom the lesion is chronic and there is fatty degeneration of the muscle, there are two possibilities: if the lesion is asymptomatic, it is better to try a non-operative treatment (54); if the lesion is painful, it is necessary to implant a TRSA.

With regard to loosening of the glenoid component it is noted that the presence of a radiolucent line around the glenoid should not have important implications. Careful preparation of the glenoid, avoiding excessive reaming, so as to preserve good bone stock, and use of pressurized cement could help to prevent subsequent mobilization. Moreover, revision surgery is not necessary if patients are completely asymptomatic.

Glenoid loosening can be the consequence of an infection, a rotator cuff tear, or both. Cheung et al. (55) showed that $30 \%$ of patients with glenoid mobilization had an underlying infection. Half of their sample underwent surgical implantation of a new component. These patients recorded better results than other half, who underwent bone grafting without glenoid reimplantation (55).

\section{References}

1. Lugli T. Artificial shoulder joint by Péan (1893): the facts of an exceptional intervention and the prosthetic method. Clin Orthop Relat Res. 1978;(133):215-218.

2. Neer CS 2nd. Displaced proximal humeral fractures. II. Treatment of three-part and four-part displacement. J Bone Joint Surg Am. 1970;52:1090-103.

3. Neer CS 2nd, Watson KC, Stanton FJ. Recent experience in total shoulder replacement. J Bone Joint Surg Am. 1982; 64:319-337.

4. Walch G, Boileau P, Noël E. Shoulder arthroplasty: evolving techniques and indications. Joint Bone Spine. 2010;77:501505 .

5. Boileau P, Sinnerton RJ, Chuinard C, Walch G. Arthroplasty of the shoulder. J Bone Joint Surg Br. 2006;88:562-575.

6. Ahearn N, McCann PA, Tasker A, Sarangi PP. The influence of rotator cuff pathology on functional outcome in total shoulder replacement. Int I Shoulder Surg. 2013;7:127-131.

7. Matsen FA 3rd, Boileau P, Walch G, Gerber C, Bicknell RT. The reverse total shoulder arthroplasty. J Bone Joint Surg Am. 2007;89:660-667.

8. Lynch JR, Franta AK, Montgomery WH Jr, Lenters TR, Mounce D, Matsen FA 3rd. Self-assessed outcome at two to four years after shoulder hemiarthroplasty with concentric glenoid reaming. J Bone Joint Surg Am. 2007;89:1284-1292.

9. Porcellini G, Abdelkhalki N, Castagna A, Campi F, Paladini P. La Spalla. Patologia, tecnica chirurgica, riabilitazione. II/Ed. Rome, Verduci Ed. 2014.

10. Edwards TB, Boulahia A, Kempf JF, Boileau P, Némoz C Walch G. Shoulder arthroplasty in patients with osteoarthritis and dysplastic glenoid morphology. J Shoulder Elbow Surg. 2004;13:1-4.

11. Matsen FA 3rd, Bicknell RT, Lippitt SB. Shoulder arthroplasty: the socket perspective. J Shoulder Elbow Surg. 2007;16 (5 Suppl):S241-S247.

12. Hettrich CM, Weldon EJ 3rd, Boorman RS, Parsons IM 4th, Matsen FA 3rd. Preoperative factors associated with improvements in shoulder function after humeral hemiarthroplasty. J Bone Joint Surg Am. 2004;86-A:1446-1451.

13. Hill JM, Norris TR. Long-term results of total shoulder arthroplasty following bone-grafting of the glenoid. J Bone Joint Surg Am. 2001;83-A:877-883.

14. Iannotti JP, Frangiamore SJ. Fate of large structural allograft for treatment of severe uncontained glenoid bone deficiency. J Shoulder Elbow Surg. 2012;21:765-71.

15. Sperling JW, Cofield RH, Steinmann SP. Shoulder arthroplasty for osteoarthritis secondary to glenoid dysplasia. J Bone Joint Surg Am. 2002;84-A:541-546.

16. Aldinger PR, Raiss P, Rickert M, Loew M. Complications in shoulder arthroplasty: an analysis of 485 cases. Int Orthop. 2010;34:517-524.

17. Bishop JY, Flatow EL. Humeral head replacement versus total shoulder arthroplasty: clinical outcomes - a review. J Shoulder Elbow Surg. 2005;14 (1 Suppl S):141S-146S.

18. Edwards TB, Kadakia NR, Boulahia A, Kempf JF, Boileau P, 
Némoz C, et al. A comparison of hemiarthroplasty and total shoulder arthroplasty in the treatment of primary glenohumeral osteoarthritis: results of a multicenter study. J Shoulder Elbow Surg. 2003;12:207-213

19. Favard L, Katz D, Colmar M, Benkalfate T, Thomazeau H, Emily S. Total shoulder arthroplasty - arthroplasty for glenohumeral arthropathies: results and complications after a minimum follow-up of 8 years according to the type of arthroplasty and etiology. Orthop Traumatol Surg Res. 2012;98 (4 Suppl): S41S47.

20. Gonzalez JF, Alami GB, Baque F, Walch G, Boileau P. Complications of unconstrained shoulder prostheses. J Shoulder Elbow Surg. 2011;20:666-682.

21. Walch G, Moraga C, Young A, Castellanos-Rosas J. Results of anatomic nonconstrained prosthesis in primary osteoarthritis with biconcave glenoid. J Shoulder Elbow Surg. 2012;21: 15261533.

22. Norris TR, Iannotti JP. Functional outcome after shoulder arthroplasty for primary osteoarthritis: a multicenter study. J Shoulder Elbow Surg. 2002;11:130-135.

23. Karelse A, Leuridan S, Van Tongel A, Piepers IM, Debeer P, De Wilde LF. A glenoid reaming study: how accurate are current reaming techniques? J Shoulder Elbow Surg. 2014;23: 11201127.

24. Walch G, Badet R, Boulahia A, Khoury A. Morphologic study of the glenoid in primary glenohumeral osteoarthritis. J Arthroplasty. 1999;14:756-760.

25. Boileau P, Avidor C, Krishnan SG, Walch G, Kempf JF, Molé D. Cemented polyethylene versus uncemented metal-backed glenoid components in total shoulder arthroplasty: a prospective, double-blind, randomized study. J Shoulder Elbow Surg. 2002;11:351-359.

26. Gerber C, Costouros JG, Sukthankar A, Fucentese SF. Static posterior humeral head subluxation and total shoulder arthroplasty. J Shoulder Elbow Surg. 2009;18:505-510.

27. Cadet ER, Ahmad CS. Hemiarthroplasty for three- and fourpart proximal humerus fractures. J Am Acad Orthop Surg. 2012;20:17-27.

28. Hertel R, Hempfing A, Stiehler M, Leunig M. Predictors of humeral head ischemia after intracapsular fracture of the proximal humerus. J Should Elbow Surg. 2004;13:427-433.

29. Jaeger M, Maier D, Izadpanah K, Strohm P, Südkamp NP. Limitations of reconstruction - prostheses. Unfallchirurg. 2011;114:1068-10674.

30. Boileau P, Krishnan SG, Tinsi L, Walch G, Coste JS, Molé D. Tuberosity malposition and migration: reasons for poor outcomes after hemiarthroplasty for displaced fractures of the proximal humerus. J Shoulder Elbow Surg. 2002;11: 401-412.

31. Robinson CM, Page RS, Hill RM, Sanders DL, Court-Brown CM, Wakefield AE. Primary hemiarthroplasty for treatment of proximal humeral fractures. J Bone Joint Surg Am. 2003; 85A:1215-1223

32. Postacchini R, Castagna A, Borroni M, Cinotti G, Postacchini F, Gumina S. Total shoulder arthroplasty for the treatment of failed hemiarthroplasty in patients with fracture of the proximal humerus. J Shoulder Elbow Surg. 2012;21:1542-1549.

33. Nijs S, Reuther F, Broos P. Primary fracture arthroplasty of the proximal humerus using a new and freely adjustable modular prosthesis combined with compression osteosynthesis of the tuberosities. Oper Orthop Traumatol. 2011;23:21-28.

34. Antuña SA, Sperling JW, Cofield RH. Shoulder hemiarthroplasty for acute fractures of the proximal humerus: a minimum five-year follow-up. J Should Elb Surg. 2008;17:202-209.

35. Levy JC, Virani N, Pupello D, Frankle M. Use of the reverse shoulder prosthesis for the treatment of failed hemiarthroplasty in patients with glenohumeral arthritis and rotator cuff deficiency. J Bone Joint Surg Br. 2007;89:189-195.

36. Flury MP, Frey P, Goldhahn J, Schwyzer HK, Simmen BR
Reverse shoulder arthroplasty as a salvage procedure for failed conventional shoulder replacement due to cuff failure-midterm results. Int Orthop. 2011;35:53-60.

37. Boileau P, Walch G. Prosthetic adaptability: a new concept in shoulder arthroplasty. In: Walch G, Boileau P (eds). Shoulder arthroplasty. Heidelberg, Springer. 1999;83-94.

38. Merolla G, Paladini P, Campi F, Porcellini G. Efficacy of anatomical prostheses in primary glenohumeral arthritis. Chir Organi Mov. 2008;91:109-115.

39. Singh JA, Sperling J, Buchbinder R, McMaken K. Surgery for shoulder osteoarthritis. Cochrane Database Syst Rev. 2010; (10): CD008089.

40. Rasmussen JV. Outcome and risk of revision following shoulder replacement in patients with glenohumeral osteoarthritis. Acta Orthop Suppl. 2014;85:1-23.

41. van den Bekerom MP, Geervliet PC, Somford MP, van den Borne MP, Boer R. Total shoulder arthroplasty versus hemiarthroplasty for glenohumeral arthritis: a systematic review of the literature at long-term follow-up. Int J Shoulder Surg. 2013;7:110-115.

42. Sperling JW, Hawkins RJ, Walch G, Zuckerman JD. Complications in total shoulder arthroplasty. J Bone Joint Surg Am. 2013; 95:563-569.

43. Kumar S, Sperling JW, Haidukewych GH, Cofield RH. Periprosthetic humeral fractures after shoulder arthroplasty. J Bone Joint Surg Am. 2004;86-A: 680-689.

44. Coste JS, Reig S, Trojani C, Berg M, Walch G, Boileau P. The management of infection in arthroplasty of the shoulder. J Bone Joint Surg Br. 2004;86:65-69.

45. Sperling JW, Kozak TK, Hanssen AD, Cofield RH. Infection after shoulder arthroplasty. Clin Orthop Relat Res. 2001;(382):206-216.

46. Ahrens P, Boileau P, Walch G. Anterior and posterior instability after unconstrained shoulder arthroplasty. In: Walch $G$, Boileau P, Molé D (eds) 2000 Shoulder Prostheses: Two to Ten Year Follow-up, Montpellier, Sauramps Medical. 2001;359-393.

47. Bohsali KI, Wirth MA, Rockwood CA Jr. Complications of total shoulder arthroplasty. J Bone Joint Surg Am. 2006;88:2279-2292.

48. Sanchez-Sotelo J, Sperling JW, Rowland CM, Cofield RH. Instability after shoulder arthroplasty: results of surgical treatment. J Bone Joint Surg Am. 2003;85-A:622-631.

49. Moeckel BH, Altchek DW, Warren RF, Wickiewicz TL, Dines DM. Instability of the shoulder after arthroplasty. J Bone Joint Surg Am. 1993;75:492-497.

50. Chin PY, Sperling JW, Cofield RH, Schleck C. Complications of total shoulder arthroplasty: are they fewer or different? J Shoulder Elbow Surg. 2006;15:19-22.

51. Cofield RH, Edgerton BC. Total shoulder arthroplasty: complications and revision surgery. Instr Course Lect. 1990;39: 449-462.

52. Deprey F. Problèmes de coiffe après prothèse d'épaule. In: Walch G, Boileau P, Molé D (eds) 2000 Shoulder Prostheses: Two to Ten Year Follow-up. Montpellier, Sauramps Medical. 2001;393-399.

53. Young AA, Walch G, Pape G, Gohlke F, Favard L. Secondary rotator cuff dysfunction following total shoulder arthroplasty for primary glenohumeral osteoarthritis: results of a multicenter study with more than five years of follow-up. J Bone Joint Surg Am. 2012;94:685-693.

54. Elhassan B, Ozbaydar M, Massimini D, Diller D, Higgins L, Warner JJP. Transfer of pectoralis major for the treatment of irreparable tears of subscapularis: does it work? J Bone Joint Surg Br. 2008;90:1059-1065.

55. Cheung EV, Sperling JW, Cofield RH. Revision shoulder arthroplasty for glenoid component loosening. J Shoulder Elbow Surg. 2008;17:371-375. 